The AstrophysiCal Journal, 317:588-592, 1987 June 15

(C) 1987. The American Astronomical Society. All rights reserved. Printed in U.S.A.

\title{
THE LOCAL EXTRAGALACTIC VELOCITY FIELD, THE LOCAL MEAN MASS DENSITY, AND BIASED GALAXY FORMATION
}

\author{
M. E. Brown and P. J. E. Peebles \\ Joseph Henry Laboratories, Princeton University \\ Received 1986 September 18; accepted 1986 November 26
}

\begin{abstract}
The biased galaxy formation picture accounts for the low apparent mass density derived from clustering dynamics by the assumption that the mass per galaxy is unusually low in the regions of high density where clustering has been studied. It would follow that the mass per galaxy is unusually high where the mass density is low, and, by continuity, that the mass per galaxy is close to the global mean in regions where the ambient mass density, $\rho_{l}$, is close to the global mean, $\rho_{b}$. That is, we would expect that the best chance for an unbiased estimate of the mean mass per galaxy, and hence of $\rho_{b}$, would be from the dynamics of regions with $\rho_{l} \approx \rho_{b}$. The local density at redshifts $200 \lesssim c z \lesssim 400 \mathrm{~km} \mathrm{~s}^{-1}$ must be close to $\rho_{b}$ because, as Sandage has emphasized, the local Hubble flow is so little perturbed. In this paper we derive a relationship between the local mass density and the perturbation of the local Hubble flow. The local mass density is estimated by the method used in the Virgocentric flow. We use the infrared Tully-Fisher distances of Aaronson et al. to find limits on the gravitational perturbation to the local Hubble flow, and we use bright galaxy counts, $N$, to estimate the local galaxy concentration. The statistics on the latter are weak because $N$ is small. We can conclude, however, that if mass were proportional to $N$, with no fluctuations, and the local mass per galaxy were a fair sample, then the density parameter $\left(\Omega=\rho_{b} /\right.$ Einstein-de Sitter density) would be $\Omega \approx 0.1$, consistent with the other dynamical estimates and inconsistent with the above naive interpretation of biasing.
\end{abstract}

Subject headings: cosmology — galaxies: clustering — galaxies: formation - galaxies: redshifts

\section{INTRODUCTION}

The ambient mass per galaxy may be defined as the ratio

$$
m_{l}=\rho_{l} / n_{l},
$$

where $\rho_{l}$ and $n_{l}$ are the mass density and the number density of bright galaxies averaged through a window that contains one or several bright galaxies. In the biased galaxy formation picture (Kaiser 1986; Davis et al. 1985, and references therein) it is argued that $m_{l}$ may be a decreasing function of increasing $\rho_{l}$. That would mean that galaxies are more tightly clustered than mass, as might happen if galaxies in low-density regions tended to be destroyed by the galaxies in denser regions that formed earlier. Since $m_{l}$ usually is estimated in systems with $\rho_{l}$ much greater than the global mass density, $\rho_{b}$, this picture would reconcile the low dynamical estimates of $m_{l}$ with the large value of the global mean mass per galaxy, $m_{b}$, implied by the Einstein-de Sitter cosmological model. Assuming $m_{l}$ is fixed by $\rho_{l}$ with little scatter, we would expect to find that $m_{l}>m_{b}$ in regions where $\rho_{l} \ll \rho_{b}$, so as to conserve mass and galaxies, and therefore that $m_{l}$ intersects $m_{b}$ where $\rho_{l}$ is close to $\rho_{b}$. An estimate of $m_{l}$ in our neighborhood, where we know $\rho_{l} \approx \rho_{b}$ (Sandage 1986), thus would be of considerable interest.

In this paper we obtain estimates of $n_{l} / n_{b}$ and $\rho_{l} / \rho_{b}$, from which we can compare $m_{l}$ and $m_{b}=\rho_{b} / n_{b}$. The estimate of $\rho_{l} / \rho_{b}$ is based on the gravitational perturbation to the local Hubble flow.

The relation between $\rho_{l} / \rho_{b}$ and the gravitational perturbation to the local Hubble flow is presented in the next section. To estimate the local Hubble flow and to obtain a count of the local number of bright galaxies we need a model for the tidal field created by the Local Supercluster. Our model, based on the analysis of the virgocentric flow (Peebles 1976; Davis and Peebles 1983), is presented in $\S$ III. In $\S$ IV the perturbation to the local Hubble flow is estimated from the infrared TullyFisher distances of Aaronson et al. (1982, hereafter A82; 1986, hereafter A86). In $\S \mathrm{V}$ we estimate the local galaxy concentration from the RSA (Sandage and Tammann 1981), KT (KraanKorteweg and Tammann 1979), and CfA (Huchra et al. 1983) catalogs.

\section{MODEL FOR THE RELATION BETWEEN THE LOCAL MASS DENSITY AND VELOCITY FIELD}

In a linear irrotational velocity field the time rate of change of the expansion is related to the mass density, $\rho_{l}$, and the shear, $\sigma^{2}=\Sigma \sigma^{a b} \sigma^{a b}$, by the equation (Peebles 1980, p. 90)

$$
\frac{d H_{l}}{d t}+H_{l}^{2}+\frac{\sigma^{2}}{3}=-\frac{4 \pi}{3} G \rho_{l}, \quad \frac{d \rho_{l}}{d t}=-3 \rho_{l} H_{l} .
$$

Because $\sigma \ll H_{l}$, the $\sigma^{2}$ term is small and so can be ignored. In this approximation we obtain the usual equations for a homogeneous isotropic cosmological model. With zero cosmological constant the local mass density and expansion rate relative to the background model are (Peebles 1980, $§ 19$ )

$$
\begin{aligned}
\frac{\rho_{l}}{\rho_{b}} & =\frac{(\sinh \theta-\theta)^{2}}{(\cosh \theta-1)^{3}} \frac{(\cosh \eta-1)^{3}}{(\sinh \eta-\eta)^{2}}, \\
\frac{H_{l}}{H_{b}} & =\frac{\sinh \theta(\sinh \theta-\theta)(\cosh \eta-1)^{2}}{\sinh \eta(\sinh \eta-\eta)(\cosh \theta-1)^{2}}, \\
\cosh \eta & =2 \Omega^{-1}-1 .
\end{aligned}
$$

The density parameter, $\Omega$, is the ratio of $\rho_{b}$ to the Einstein-de Sitter mass density. Given $\rho_{l} / \rho_{b}$, these equations fix $H_{l} / H_{b}$ as a function of $\Omega$, as shown in Figure 1 .

We will compare $\rho_{l} / \rho_{b}$ to the local count of bright galaxies relative to what would be expected for a homogeneous dis- 


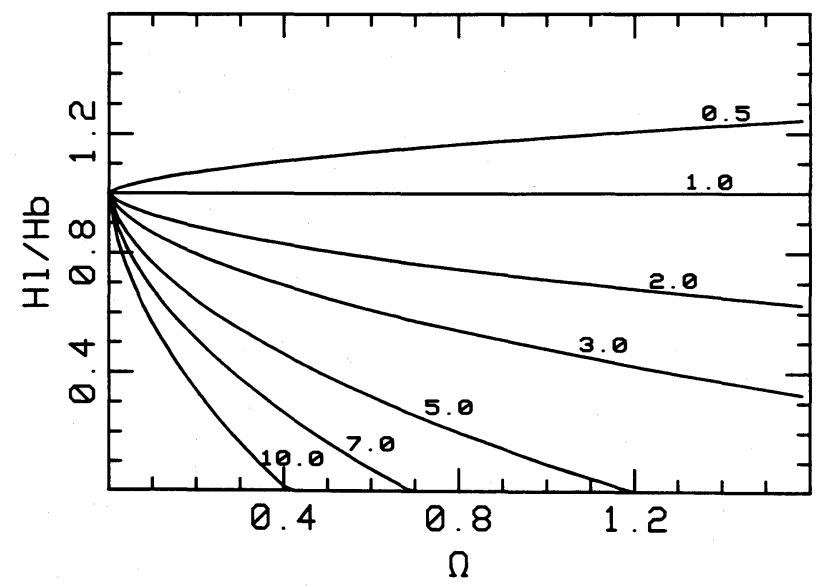

FIG. 1.-Local expansion rate as a function of cosmological density parameter, $\Omega$, for given local density, $\rho_{\nu} / \rho_{b}$ (eqs. [3]). The curves are labeled by the value of $\rho_{l} / \rho_{b}$.

tribution. Another way to proceed would be to compare $\rho_{l} / \rho_{b}$ to the ratio of luminosity densities,

$$
\Delta=j_{l} / j_{b} .
$$

If $\Delta$ were estimated assuming the expansion rate everywhere is $H_{b}$, and if the true luminosity density were proportional to the mass density, then $\Delta$ would be given by the equation

$$
\Delta=\frac{\rho_{l}}{\rho_{b}} \frac{H_{b}}{H_{l}} .
$$

The relation between $H_{l} / H_{b}$ and $\Omega$ for given $\Delta$ is shown in Figure 2.

III. MODEL FOR THE LOCAL VELOCITY FIELD

To estimate $H_{l}$ and the local concentration of galaxies we need a model for the effect of the tidal field of the Local Supercluster on the local velocity field. We model this as a linear function of distance, with an isotropic term to represent the local expansion rate, $H_{l}$, and a shear term to represent the tidal field of the Local Supercluster. As a guide to the latter we use the model in which the radial virgocentric flow varies with distance $D$ from the Virgo cluster as $D^{-n}$, with $n \approx 1$ (Davis

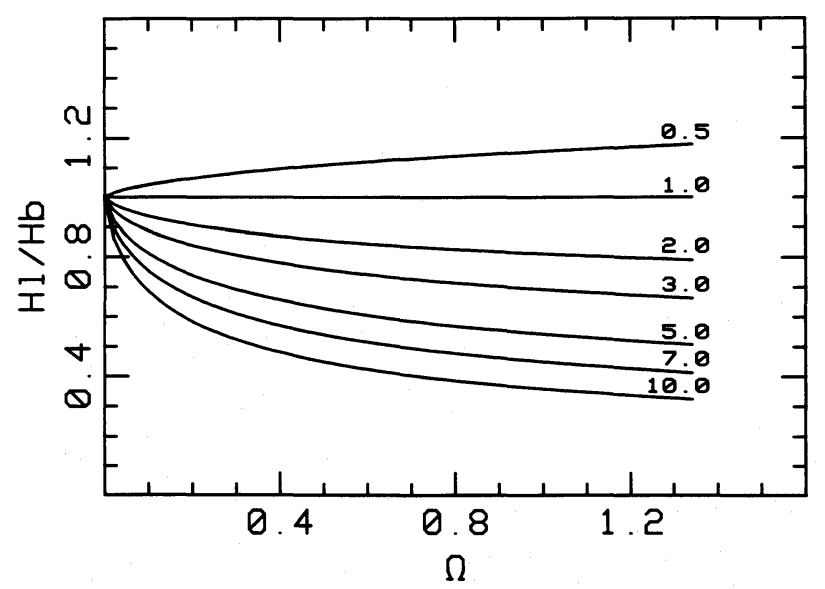

FIG. 2.-Local exapansion rate as a function of $\Omega$ for fixed apparent local luminosity density ratio, $\Delta$ (eqs. [4], [5]). The curves are labeled by the value of $\Delta$. and Peebles 1983). In this model the local relative velocity field is, to first order in distance, $r$, from us,

$$
\begin{gathered}
v^{a}=H_{l} r^{a}+\sum_{b} \sigma^{a b} r_{b}, \\
\sigma_{x x}=-2 \sigma_{y y}=-2 \sigma_{z z}=\frac{2}{3}(n+1) v_{v} / R,
\end{gathered}
$$

where $v_{v} \approx 200 \mathrm{~km} \mathrm{~s}^{-1}$ is our peculiar velocity toward the Virgo Cluster (in the $x$-direction), our distance from the Virgo cluster is

$$
R=\left(v_{v}+1000 \mathrm{~km} \mathrm{~s}^{-1}\right) / H_{b},
$$

where the redshift of the Virgo Cluster is $\sim 1000 \mathrm{~km} \mathrm{~s}^{-1}$, and $H_{l}$ and $H_{b}$ are the local and global expansion rates, with

$$
\frac{H_{l}}{H_{b}}=1-\frac{(2-n)}{3} \frac{v_{v}}{H_{b} R} .
$$

Our model for the local velocity field will be equation (6) with $H_{l}$ a free parameter, to take account of the local mass density, and with the tidal term fixed by $n=1$ and $v_{v}$ in the range $0-300 \mathrm{~km} \mathrm{~s}^{-1}$. Any shear field in this range about equally well fits the data (Fig. 3 below).

\section{ESTIMATES OF THE LOCAL EXPANSION RATE}

The local expansion rate, $H_{l}$, has been estimated by Sandage (1986). For an independent check, we use the infrared TullyFisher distance measures of A82 as calibrated by A86. The calibration uses 10 clusters at $c z \approx 4000-10,000 \mathrm{~km} \mathrm{~s}^{-1}$ plus the Virgo Cluster, where $c z$ is the true distance multiplied by $H_{b}$. The redshift of the Virgo Cluster is corrected for Virgocentric flow, and the other redshifts are corrected for our motion relative to the microwave background. If other peculiar motions are small or average out, the calibration yields distances in units of the cosmological redshift, which, as A86 emphasize, does not depend on the absolute distance scale. The result is

$$
\begin{aligned}
\log c z & =1.174+0.2 H+2.236 X-1.50 X^{2}, \\
X & =\log \Delta v-2.5,
\end{aligned}
$$

\begin{tabular}{|c|c|c|c|}
\hline Galaxy & $c z^{\mathrm{a}}$ & $v_{0}^{\mathrm{b}}$ & $\cos \theta$ \\
\hline $\mathrm{N} 247$ & 237 & 227 & -0.99 \\
\hline N253 & 256 & 293 & -0.97 \\
\hline N2403.. & 260 & 240 & 0.31 \\
\hline N3031.. & 300 & 240 & 0.47 \\
\hline N3621.......... & 505 & 435 & 0.66 \\
\hline N4236............ & 302 & 240 & 0.54 \\
\hline N4244.... & 342 & 249 & 0.90 \\
\hline $\mathrm{N} 4258 \ldots$ & 533 & 520 & 0.82 \\
\hline N4826.......... & 335 & 350 & 0.98 \\
\hline N5585.......... & 560 & 368 & 0.66 \\
\hline N7793 ........... & 306 & 241 & -0.93 \\
\hline
\end{tabular}

where $H$ and $\Delta v$ are the A82 infrared magnitude and $21 \mathrm{~cm}$ line width.

Table 1 lists all galaxies, with $\Delta v$ and $H$ in A82, that are outside the Local Group, that have $200<\Delta v<560$, as recommended by A86, and that have $v_{0}$ and $c z<600 \mathrm{~km} \mathrm{~s}^{-1}$, where $v_{0}$ is the observed redshift velocity.

TABLE 1

a Eq. (9).

b Corrected observed redshift, $\mathrm{km} \mathrm{s}^{-1}$. 
TABLE 2

\begin{tabular}{|c|c|c|c|}
\hline \multicolumn{4}{|c|}{ Estimates of $H_{l} / H_{b}$} \\
\hline \multirow[b]{2}{*}{$\begin{array}{l}v_{v} \\
\text { (1) }\end{array}$} & \multicolumn{3}{|c|}{$v_{\max }$} \\
\hline & $\begin{array}{c}300 \\
(2)\end{array}$ & $\begin{array}{c}400 \\
(3)\end{array}$ & $\begin{array}{c}600 \\
(4)\end{array}$ \\
\hline 0. & 0.95 & 0.89 & 0.86 \\
\hline $150 .$. & 0.90 & 0.81 & 0.80 \\
\hline 300. & 0.86 & 0.75 & 0.74 \\
\hline
\end{tabular}

The observed redshifts, $v_{0}$, corrected for motion in the Local Group, are taken from RSA. Where RSA assign the galaxy to a group other than $\mathrm{S}$ we use the RSA redshift for the group. We use individual redshifts for the $S$ group members because RSA notes the large spread in apparent distances of these galaxies. In the last column in the table, $\theta$ is the angular distance from the galaxy to the Virgo Cluster.

The model in equation (6) for the local velocity field gives

$$
v_{0}-\kappa c z\left(\cos ^{2} \theta-\frac{1}{3}\right)=c z H_{l} / H_{b},
$$

where the amplitude of the tidal term is taken to be

$$
\kappa=2 v_{v} /\left(1000+v_{v}\right),
$$

with $n \approx 1$. We could estimate $H_{l} / H_{b}$ by dividing equation (10) by $c z$ and then averaging the left side over the sample. However, that would introduce a bias if measurement error made any of the estimates of $z$ close to zero. Therefore we estimate $H_{l} / H_{b}$ as the ratio of the mean value of the left side of equation (10) to the mean value of $c z$. The sample over which we average is the set of galaxies from Table 1 with $v_{0}<v_{\max }$ and $c z<v_{\max }$, and the average is computed for a fixed assumed value of the tide parameter, $\kappa$. Table 2 lists the results for three choices of the velocity cutoff, $v_{\max }$, and three choices of $\kappa$. At the three choices of $v_{\max }$ there are four, eight, and 11 galaxies. We adopt the results in column (3) for $v_{\max }=400$ as the best compromise between a local measure and a reasonable number of galaxies.

Figure 3 shows the ratios $v_{0} / c z$ plotted against $\cos ^{2} \theta-\frac{1}{3}$. The lines are given by equation (10) with the parameters in column (3) of Table 2. (These lines may not appear to be quite the best fits because the distribution of errors in $v_{0} / c z$ is skewed by the division by $c z$.) We conclude from the figure that the tidal field is at best marginally detected in the data and that the three cases in column (3) of Table 2 are about equally good representations of the data.

The model in equation (8) with $v_{v}=300$ gives $H_{l} / H_{b} \approx 0.92$, which is well above the results in the last row of Table 2 . This difference would say that the local mass density is larger than the density average over a spherical shell at our distance from the Virgo Cluster.

Sandage's (1986) analysis can be compared to the first row of Table 2, and would indicate bounds on $H_{l} / H_{b}$ that are even closer to unity. In the following we use the estimates in Table 2 , but we note that increasing $H_{l} / H_{b}$ exacerbates the problem of reconciling the galaxy concentration with a dense cosmological model.

\section{ESTIMATE OF THE LOCAL GALAXY DENSITY}

In this section we estimate the ratio $n_{l} / n_{b}$ of local to global mean densities of galaxies from the counts of galaxies brighter than CfA magnitude - 19.0. We choose this limit because the luminosity function derived by Davis and Huchra (1982, hereafter DH) from the CfA catalog shows very little scatter between north and south subsets at brighter magnitudes. The integral of the DH luminosity function to $M=-19$ is

$$
\phi(<-19)=0.0044 \mathrm{Mpc}^{-3} \text {. }
$$

The expected number of galaxies at cosmological redshifts $<c z$ for a homogeneous distribution is then

$$
N_{e}=\frac{4 \pi}{3}\left(\frac{c z}{100}\right)^{3} \phi=0.0184\left(\frac{c z}{100}\right)^{3} \text {. }
$$

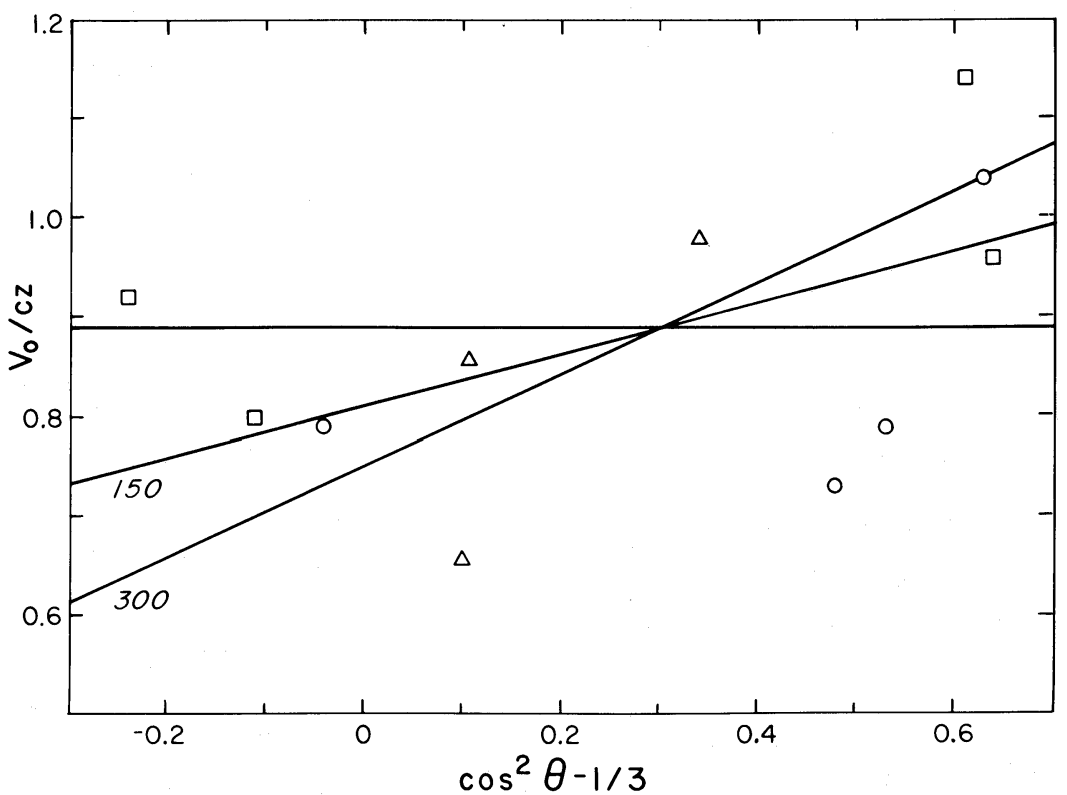

Fig. 3.-Ratio of observed to cosmological redshift as a function of angular distance, $\theta$, from the Virgo Cluster. Squares are galaxies at $c z<300 \mathrm{~km} \mathrm{~s}^{-1}$, circles $300<c z<400$, and triangles, $400<c z<600$. Lines are the model (eq. [10]) with parameters from col. (3) of Table 2 . The horizontal line has no tidal field. The slanted lines have tidal components with $\kappa$ given by eq. (11). 
TABLE 3

Bright Nearby Galaxies

\begin{tabular}{|c|c|c|c|c|c|}
\hline Galaxy & $l$ & $b$ & $M_{\mathrm{RSA}}+A^{i}$ & $v_{0}$ & $\cos \theta$ \\
\hline N253 & 98 & -88 & -20.71 & 293 & -0.97 \\
\hline MI ... & 136 & -1 & -20.5 & 234 & -0.24 \\
\hline MII . & 137 & 0 & -20.5 & 234 & -0.22 \\
\hline$\ldots \ldots \ldots$ & 138 & 11 & -20.45 & 234 & -0.03 \\
\hline N3031 .. & 142 & 41 & -19.97 & 240 & 0.47 \\
\hline N4736 .......... & 123 & 76 & -20.27 & 345 & 0.87 \\
\hline N5128 .......... & 310 & 19 & -21.6 & 255 & 0.54 \\
\hline N5236 .......... & 315 & 32 & -20.81 & 255 & 0.70 \\
\hline N5457 ........... & 102 & 60 & -21.22 & 368 & 0.70 \\
\hline N6946 .......... & 96 & 12 & -19.98 & 336 & -0.06 \\
\hline
\end{tabular}

We make no correction for bright galaxies lost in the Milky Way. Any such galaxies would add to the local concentration and so increase the problem with $\Omega=1$.

For the local galaxy count we use RSA supplemented by KT for galaxies not in RSA. We transform from RSA magnitudes by the equation

$$
M=M_{\mathrm{RSA}}+A^{i}+0.18+5 \log 2 v_{0} / c z .
$$

The first term on the right-hand side is the RSA absolute magnitude (with the exception of the $S$ group; where we compute $M$ from $v_{0}$ as in the last section). The next term removes the correction for internal absorption, to be consistent with CfA. The next term is the mean difference of apparent magnitudes of the 11 galaxies in Table 3 for which we have RSA and CfA magnitudes. The factor of 2 in the logarithm brings the distance scale to the nominal value $H_{b}=100 \mathrm{~km} \mathrm{~s}^{-1} \mathrm{Mpc}^{-1}$ in $\mathrm{DH}$. The last factor is our model for $c z / v_{0}$ (eqs. [10], [11]); this corrects $M$ to the "true" distance $\propto z$. We use the RSA corrections for galactic absorption, which is consistent with $\mathrm{DH}$.

Given the parameters $H_{l} / H_{b}$ and $v_{v}$, equations (10), (11), and (14) fix the translation from $M_{\mathrm{RSA}}$ and $v_{0}$ to absolute magnitude $M$ and true distance $c z / H_{b}$. Table 3 lists the galaxies in RSA or KT for which $M<-19$ and $c z<500$ for any of the choices of parameters in Table 4.

Column (4) in Table 4 lists the counts $N$ of galaxies at $M<-19$ and at redshift distances of $c z<400$ and $c z<500$ $\mathrm{km} \mathrm{s}^{-1}$. The count always includes the Milky Way and the Andromeda nebula. The first three entries assume no tidal field ( $\kappa=0)$. The second value of $H_{l} / H_{b}$ is the estimate in column (3)

TABLE 4

Local Galaxy Counts and Mass Densities

\begin{tabular}{|c|c|c|c|c|c|c|c|}
\hline \multirow[b]{2}{*}{$\begin{array}{c}H_{l} / H_{b} \\
\quad(1)\end{array}$} & \multirow[b]{2}{*}{$\begin{array}{l}v_{v} \\
(2)\end{array}$} & \multirow[b]{2}{*}{$c z_{\max }$} & \multirow[b]{2}{*}{$\begin{array}{l}N \\
(4)\end{array}$} & \multicolumn{2}{|c|}{$N_{e}^{\prime \mathrm{a}}$} & \multicolumn{2}{|c|}{$\rho_{l} / \rho_{b}(\Omega=1)$} \\
\hline & & & & $\begin{array}{c}\Omega=1 \\
(5)\end{array}$ & $\begin{array}{c}\Omega=0.1 \\
(6)\end{array}$ & $\begin{array}{c}z=0 \\
(7)\end{array}$ & $\begin{array}{c}z=4 \\
(8)\end{array}$ \\
\hline $1.0 \ldots$ & 0 & $\begin{array}{l}400 \\
500\end{array}$ & $\begin{array}{l}6 \\
6\end{array}$ & $\begin{array}{l}1.2 \\
2.3\end{array}$ & $\begin{array}{l}1.2 \\
2.3\end{array}$ & 1.00 & 1.00 \\
\hline $0.89 \ldots \ldots \ldots$ & 0 & $\begin{array}{l}400 \\
500\end{array}$ & $\begin{array}{l}8 \\
9\end{array}$ & $\begin{array}{l}1.6 \\
3.1\end{array}$ & $\begin{array}{l}3.1 \\
6.0\end{array}$ & 1.35 & 1.06 \\
\hline $0.75 \ldots \ldots \ldots$ & 0 & $\begin{array}{l}400 \\
500\end{array}$ & $\begin{array}{r}9 \\
11\end{array}$ & $\begin{array}{l}2.2 \\
4.3\end{array}$ & $\begin{array}{r}6.3 \\
12.3\end{array}$ & 1.85 & 1.11 \\
\hline $0.81 \ldots \ldots \ldots$ & 150 & $\begin{array}{l}400 \\
500\end{array}$ & $\begin{array}{r}8 \\
10\end{array}$ & $\begin{array}{l}1.9 \\
3.8\end{array}$ & $\begin{array}{l}4.8 \\
9.4\end{array}$ & 1.63 & 1.09 \\
\hline $0.75 \ldots \ldots \ldots$ & 300 & $\begin{array}{l}400 \\
500\end{array}$ & $\begin{array}{l}10 \\
11\end{array}$ & $\begin{array}{l}2.2 \\
4.3\end{array}$ & $\begin{array}{r}6.3 \\
12.3\end{array}$ & 1.85 & 1.11 \\
\hline
\end{tabular}

${ }^{\text {a }}$ Eq. (20). of Table 2; the third, a conservative lower bound on $H_{l} / H_{b}$ from the A82, A86 data. The last two entries are the fits to the tidal field model in column (3) of Table 2.

We can compare $N$ to several numbers. First, if the galaxy distribution were a homogeneous random process the expected counts (corrected for the bias that we are in a galaxy) would be (eq. [13])

$$
\begin{aligned}
1+N_{e} & =2.2(c z<400), \\
& =3.3(c z<500) .
\end{aligned}
$$

The observed $N$ is about 3-8 times $1+N_{e}$. Second, to estimate the local mean number density of galaxies we might subtract 2 from $N$ to remove the bias from the fact that we are in a group with two bright members. That would give

$$
\frac{n_{l}}{n_{b}} \approx \frac{N-2}{N_{e}} \approx 2-8 \text {. }
$$

This can be compared to the mean density of galaxies within our position in the Local Supercluster (Yahil 1981; DH),

$$
\langle n\rangle_{\mathrm{LSC}} / n_{b} \approx 3 \text { to } 4,
$$

a roughly similar value. Third, the expected number of galaxies within distance $c z / H_{b}$ of a galaxy, taking account of the galaxy two-point correlation function $\xi(r)$ (Groth and Peebles 1986), is

$$
\begin{aligned}
N_{1} & =1+\phi \int_{0}^{r} \xi d V=1+\frac{3}{3-\gamma} N_{e}\left(\frac{H_{b} r_{0}}{c z}\right)^{\gamma}, \\
\gamma & =1.77, \quad H_{b} r_{0}=540 \mathrm{~km} \mathrm{~s}^{-1} .
\end{aligned}
$$

This gives

$$
\begin{aligned}
N_{1} & =5.9, & c z<400 \\
& =7.4, & c z<500 .
\end{aligned}
$$

These are reasonably similar to the numbers in column (4) of Table 4.

\section{COMPARISON OF THE LOCAL CONCENTRATIONS OF MASS AND GALAXIES}

In comparing the local concentrations of mass and of galaxies we should of course consider the full count, $N$, of bright galaxies because $H_{l} / H_{b}$ depends on the net local mass excess. If we assume $m_{l}=m_{b}$, then we expect the number of galaxies corrected for the local mass concentration to be

$$
N_{e}{ }^{\prime}=N_{e} \rho_{l} / \rho_{b},
$$

where $\rho_{l} / \rho_{b}$ is fixed by $\Omega$ and $H_{l} / H_{b}$ (Fig. 1). Columns (5) and (6) of Table 4 list $N_{e}^{\prime}$ for two choices of $\Omega$. We see that if $\Omega=1$ the observed count, $N$, is larger than the count expected from dynamics, $N_{e}^{\prime}$, by a factor of $\sim 4$ at $c z<400$, and by a factor $\sim 3$ at $c z<500$. If $\Omega=0.1$, and $H_{l} / H_{b} \approx 0.75-0.8, N$ and $N_{e}{ }^{\prime}$ are tolerably consistent.

Put another way, our estimate of the local galaxy number density relative to the background is

$$
\begin{aligned}
\frac{n_{l}}{n_{b}} \approx \frac{N}{N_{e}} & \approx 7, \quad c z<400 \\
& \approx 4, \quad c z<500 .
\end{aligned}
$$

The local mass density ratio is

$$
\begin{aligned}
& \rho_{l} / \rho_{b} \approx 1.6 \pm 0.3 \text { if } \Omega=1 ; \\
& \approx 3 \text { to } 5 \quad \text { if } \Omega=0.1 \text {. }
\end{aligned}
$$


If $\Omega=1$ then $\rho_{l}$ is nearly equal to $\rho_{b}$, and, following the argument in the Introduction, we might expect therefore that $m_{l} \approx m_{b}$, that is, $n_{l} / n_{b} \approx \rho_{l} / \rho_{b}$. We see, however, that that is a factor $\sim 4$ off the counts at $c z<400$. This indicates that we need biasing even though the local mass density is not very high.

Column (8) of Table 4 shows the density ratio extrapolated back to redshift $z=4$ assuming $\Omega=1$. We expect that the sites of galaxy formation were fixed by $z=4$. At this epoch the local mass density would have been $\lesssim 10 \%$ higher than the background if $\Omega=1$. This does not allow a very substantial gravitational trigger for biasing.

\section{DISCUSSION}

The central point of this paper is that, as Sandage (1986) emphasizes, the local velocity field is remarkably close to the general recession. If conventional gravity physics is correct, this means either that the mean mass density is low or else that the local mass density is quite close to the mean despite the local concentration of galaxies at $c z \lesssim 500 \mathrm{~km} \mathrm{~s}^{-1}$.

Our analysis depends on three main steps: the estimate of the local expansion rate relative to the background, $H_{l} / H_{b}$; the computation of the local mean mass density relative to the background, $\rho_{l} / \rho_{b}$ implied by $H_{l} / H_{b}$; and the estimate of the local number density of galaxies relative to the background, $n_{l} / n_{b}$. The redshift calibration of the IRTF relation may be biased by large-scale velocity fields (as reviewed by Silk 1986), but a peculiar velocity of $700 \mathrm{~km} \mathrm{~s}^{-1}$ would be only $\sim 10 \%$ of the typical redshift of an A86 cluster, and as this error may be expected to average out among the A86 clusters the effect on $H_{l} / H_{b}$ seems negligible. Our estimate of $H_{l} / H_{b}$ is based on only 11 galaxies, but the scatter (Fig. 3) is small so the result seems believable. Our use of the homogeneous cosmological model to estimate $\rho_{l} / \rho_{b}$ may seem questionable in view of the decidedly clumpy distribution of galaxies at $c z<500 \mathrm{~km} \mathrm{~s}^{-1}$. However, as noted above, we can argue that the homogeneous model really is a useful approximation because the local Hubble flow is so smooth, which tells us either that the flow has not been perturbed or else that it has been perturbed by a mass distribution that is smooth on scales $H_{b} r \lesssim 500 \mathrm{~km} \mathrm{~s}^{-1}$. Our estimate of $n_{l} / n_{b}$ depends on only $N \approx 10$ bright galaxies. While the 10 galaxies undoubtedly exist, and the estimate $n_{l} / n_{b} \approx 7$ at $c z<400$ seems reasonable in view of the general concentration of galaxies around the Local Supercluster, the small value of $N$ does leave open the possibility of a statistical interpretation, that $\rho_{l}$ and $m_{l}$ are indeed correlated, and that where $\rho_{l} \approx \rho_{b}$, $m_{l}=m_{b}$ on the average, but that the correlation is only statistical and we happen to be in a region of upward fluctuation of galaxy numbers relative to mass.

The straightforward interpretation of the results in Table 4 is that $\Omega \approx 0.1$. This is comparable to the results of all the other dynamical tests of $\Omega$ (Peebles 1986), but, as discussed in the Introduction, with the important difference that the density here is much closer to the mean so it is reasonable to expect the sample is less biased.

This straightforward interpretation conflicts with the global measure of Loh annd Spillar (1986) and with the general belief that $\Omega$ ought to be unity. If $\Omega=1$ we have an interesting constraint on the way galaxies were assembled. If the local concentration of galaxies were produced by transporting material, as in the explosion model (Ostriker and Cowie 1981), it would have to be done without perturbing the net mass density at redshift $z=4$ by more than $\sim 10 \%$ (col. [8] of Table 4). That would be possible if only baryons with density well below the total were transported, but still it seems to be a very delicate arrangement. Of course, the constraint is considerably eased if $\Omega \approx 0.1$, because the gravitational instability is suppressed. In the more standard biased galaxy formation picture, one imagines that mass clouds similar to galaxies exist (or existed) in about equal numbers everywhere and that luminosities are high enough to make the clouds recognizable as galaxies only in islands like the Local Group. There are two puzzles here. First, it is not apparent why luminosities should have been high, even on a statistical basis, in our neighborhood, since the mass density is so close to the mean. Second, if most of the local mass were in dark galaxies, it is not clear how ordinary gravitational instabilities avoided introducing considerable noise in the local Hubble flow.

We thank Dick Bond, John Huchra, Bob Schommer, and Scott Tremaine for stimulating comments. This research was supported in part by the National Science Foundation.

\section{REFERENCES}

Aaronson, M., Bothun, G., Mould, J. R., Huchra, J., Schommer, R. A., and Cornell, M. E. 1986, Ap. J., 302, 536 (A86).

Aaronson, M., et al. 1982, Ap. J. Suppl., 50, 241 (A82).

Davis, M., Efstathiou, G., Frenk, C. S., and White, S. D. M. 1985, Ap. J., 292, 371 .

Davis, M., and Huchra, J. 1982, Ap. J., 254, 437 (DH).

Davis, M., and Peebles, P. J. E. 1983, Ann. Rev. Astr. Ap., 21, 109.

Groth, E. J., and Peebles, P. J. E. 1986, Ap. J., 310, 499 .

Huchra, J., Davis, M., Latham, D., and Tonry, J. 1983, Ap. J. Suppl., 53, 89 (CfA).

Kaiser, N. 1986, in Inner Space/Outer Space, ed. E. W. Kolb, M. S. Turner, K. Olive, D. Seckel, and D. Lindley (Chicago: University of Chicago Press), p. 258.

Kraan-Korteweg, R. C., and Tammann, G. A. 1979, Astr. Nach., 300, 181 (KT). Loh, E. D., and Spillar, E. J. 1986, Ap. J. (Letters), 307, L1.

Ostriker, J. P.; and Cowie, L. L. 1981, Ap. J. (Letters), 243, L127.

Peebles, P. J. E. 1976, Ap. J., 205, 318.

Peebles, 1980, The Large-Scale Structure of the Universe (Princeton: Princeton University Press).

. 1986, Nature, 321, 27.

Sandage, A. R. 1986, Ap.J., 307, 1.

Sandage, A. R., and Tammann, G. A. 1981, A Revised Shapley-Ames Catalog of Bright Galaxies (Washington, D. C.: Carnegie Institution)(RSA).

Silk, J. 1986, Nature, 322, 207. 